\title{
The overdensities of galaxy environments as a function of luminosity and color
}

\author{
David W. Hogg ${ }^{1,2}$, Michael R. Blanton ${ }^{1}$, Daniel J. Eisenstein ${ }^{3}$, James E. Gunn ${ }^{4}$, \\ David J. Schlegel ${ }^{4}$, Idit Zehavi ${ }^{5,6}$, Neta A. Bahcall ${ }^{4}$, Jon Brinkmann ${ }^{7}$, Istvan Csabai ${ }^{8,9}$, \\ Donald P. Schneider ${ }^{10}$, David H. Weinberg ${ }^{11}$, Donald G. York ${ }^{6}$
}

\begin{abstract}
We study the mean environments of galaxies in the Sloan Digital Sky Survey as a function of rest-frame luminosity and color. Overdensities in galaxy number are estimated in $8 h^{-1} \mathrm{Mpc}$ and $1 h^{-1} \mathrm{Mpc}$ spheres centered on 125,000 galaxies taken from the SDSS spectroscopic sample. We find that, at constant color, overdensity is independent of luminosity for galaxies with the blue colors of spirals. This suggests that, at fixed star-formation history, spiral-galaxy mass is a very weak function of environment. Overdensity does depend on luminosity for galaxies with the red colors of early types; both low-luminosity and high-luminosity red galaxies are found to be in highly overdense regions.
\end{abstract}

Subject headings: galaxies: clusters: general — galaxies: fundamental parameters — galaxies: statistics — large-scale structure of universe

\footnotetext{
${ }^{1}$ Center for Cosmology and Particle Physics, Department of Physics, New York University, 4 Washington Place, New York, NY 10003

2 david.hogg@nyu.edu

${ }^{3}$ Steward Observatory, 933 N. Cherry Ave., Tucson, AZ 85721

${ }^{4}$ Princeton University Observatory, Princeton, NJ 08544

${ }^{5}$ Fermi National Accelerator Laboratory, PO Box 500, Batavia, IL 60510

${ }^{6}$ Astronomy and Astrophysics Department, University of Chicago, Chicago, IL 60637

${ }^{7}$ Apache Point Observatory, 2001 Apache Point Road, P.O. Box 59, Sunspot, NM 88349-0059

${ }^{8}$ Department of Physics, Eötvös University, Budapest, Pf. 32, Hungary, H-1518

${ }^{9}$ Department of Physics and Astronomy, The Johns Hopkins University, Baltimore, MD 21218

10 Department of Astronomy and Astrophysics, The Pennsylvania State University, University Park, PA 16802

${ }^{11}$ Department of Astronomy, Ohio State University, Columbus, OH 43210
} 


\section{Introduction}

Elliptical and lenticular galaxies are over-represented in massive nearby galaxy clusters relative to the field (Dressler 1980; Postman \& Geller 1984). Elliptical galaxies also tend to be redder, more luminous, more metal-rich, more gas-poor, and older in stellar population than spirals and irregulars (e.g., Tammann et al. 1979; Kormendy \& Djorgovski 1989; Roberts \& Haynes 1994). Indeed, it has also been found that color, luminosity, surface-brightness, gas content, stellar population age, and star-formation rate are all correlated with the overdensity

of the galaxy environment (e.g., Kennicutt 1983; Balogh et al. 2001; Martínez et al. 2002; Lewis et al. 2002; Blanton et al. 2002c; Gomez et al. 2002). Along the same lines, studies of the clustering of galaxies have found different clustering amplitudes for galaxies of different types, colors, and luminosities (e.g., Davis \& Geller 1976; Mo et al. 1994; Park et al. 1994; Norberg et al. 2002; Zehavi et al. 2002). What is not understood is which of the relationships with environment are causal and which are just a by-product of other, more fundamental correlations.

Conventional cosmological theories posit that galaxies reside inside dark matter concentrations or "halos" that grow from small random fluctuations at early times. The most overdense fluctuations will collapse first; in a gaussian random field, these preferentially reside within overdensities on larger scales, implying a correlation of halo mass with environment is expected (e.g., Mo \& White 1996; Lemson \& Kauffmann 1999). The relationships between the properties of a halo and the properties of the galaxy or galaxies it contains are not fully understood, so the conventional theories do not currently make strong predictions for the dependence of galaxy number overdensity on observable galaxy properties, although most studies suggest that luminosity and color will be related to overdensity (eg, Kauffmann et al. 1997, 1999; Benson et al. 2000). Whether or not this dark-matter halo picture ends up being useful or correct, and whatever turn out to be the important physical processes for making galaxies, the investigations started in this Letter will place important constraints on galaxy formation and evolution.

The Sloan Digital Sky Survey (SDSS) is the best available data set for investigation of these relationships because of its sample size, high signal-to-noise imaging, sky coverage, and complete spectroscopy (e.g., York et al. 2000). Indeed, the SDSS has already made some relevant measurements, including the dependence of clustering on luminosity and color (Zehavi et al. 2002), the star-formation as a function of environment (Gomez et al. 2002), and the mean red-galaxy spectrum as a function of environment (Eisenstein et al. 2002). In this Letter, we investigate the mean galaxy number overdensities around galaxies of different colors and luminosities.

In what follows, a cosmological world model with $\left(\Omega_{\mathrm{M}}, \Omega_{\Lambda}\right)=(0.3,0.7)$ is adopted, 
and the Hubble constant is parameterized $H_{0}=100 h \mathrm{~km} \mathrm{~s}^{-1} \mathrm{Mpc}^{-1}$, for the purposes of calculating distances and volumes (e.g., Hogg 1999).

\section{Data sample}

The SDSS is taking ugriz CCD imaging of $10^{4} \mathrm{deg}^{2}$ of the Northern Galactic sky, and, from that imaging, selecting $10^{6}$ targets for spectroscopy, most of them galaxies with $r<17.77$ mag (e.g., Gunn et al. 1998; York et al. 2000; Stoughton et al. 2002).

All the data processing: astrometry (Pier et al. 2002); source identification, deblending and photometry (Lupton et al. 2001); calibration (Fukugita et al. 1996; Smith et al. 2002); spectroscopic target selection (Eisenstein et al. 2001; Strauss et al. 2002; Richards et al. 2002); spectroscopic fiber placement (Blanton et al. 2002b); and spectroscopic data reduction are performed with automated SDSS software.

Redshifts are measured on the reduced spectra by an automated system, which models each galaxy spectrum as a linear combination of stellar populations (Schlegel, in preparation).

The sample is statistically complete, with small incompletenesses coming primarily from (1) galaxies missed because of mechanical spectrograph constraints (6 percent; Blanton et al. 2002b), which does lead to a slight under-representation of high-density regions, and (2) spectra in which the redshift is either incorrect or impossible to determine $(<1$ percent). In addition, there are some galaxies ( $\sim 1$ percent) blotted out by bright Galactic stars, but this incompleteness should be uncorrelated with galaxy properties.

For the purposes of computing large-scale structure statistics, we have assembled a subsample of SDSS galaxies known as the NYU LSS sample10. This subsample includes not only properties of the galaxies but also of survey selection function variations and angular coverage. For each galaxy in sample10, the sample includes a computed volume $V_{\max }$ representing the total volume of the Universe (in $h^{-3} \mathrm{Mpc}^{3}$ ) in which the galaxy could have resided and still made it into the sample. The calculation of these volumes is described elsewhere (Blanton et al. 2002c).

Galaxy luminosities and colors (measured by the standard SDSS petrosian technique; Petrosian 1976) are computed in fixed bandpasses, using Galactic extinction corrections (Schlegel et al. 1998) and $K$ corrections (computed with kcorrect v1_11; Blanton et al. 2002a). They are $K$ corrected not to the redshift $z=0$ observed bandpasses but to bluer

bandpasses ${ }^{0.1} g,{ }^{0.1} r$ and ${ }^{0.1} i$ "made" by shifting the SDSS $g, r$, and $i$ bandpasses to shorter wavelengths by a factor of 1.1 (c.f., Blanton et al. 2002a,c). This means that galaxies at 
redshift $z=0.1$ (typical of the SDSS sample used here) have trivial $K$ corrections.

The sample of galaxies used here was selected to have apparent magnitude in the range $14.5<r<17.77 \mathrm{mag}$, redshift in the range $0.05<z<0.22$, and fixed-frame absolute magnitude in the range $M_{0.1_{i}}>-24.0 \mathrm{mag}$. These cuts left 124884 galaxies.

Overdensity estimators on two different length scales are used. On the $8 h^{-1} \mathrm{Mpc}$ scale, the estimate of environment overdensity $\delta_{8}$ is based on the SDSS spectroscopic sample. It is a measure of the three-dimensional redshift-angle space number density excess around each galaxy. The comoving transverse distances and comoving line-of-sight distances (e.g., Hogg 1999) are computed between each spectroscopic galaxy and its neighboring spectroscopic galaxies (not attempting to correct for peculiar velocities). Neighbors within an $8 h^{-1} \mathrm{Mpc}$ comoving sphere in this space are counted; the result is divided by the prediction made from the galaxy luminosity function (Blanton et al. 2002d), and unity is subtracted to produce the overdensity estimate $\delta_{8}$. A galaxy in an environment with the cosmic mean density has $\delta_{8}=0$. Although the sample used to infer $\delta_{8}$ is flux-limited and not volume-limited, the resulting overdensity estimates have been shown to be redshift-independent in the median (Blanton et al. 2002c). If the spatial correlation function $\xi(r)$ has the form $r^{-\gamma}$, then the mean overdensity around galaxies $\left\langle\delta_{8}\right\rangle$ will be $(3 /[3-\gamma]) \xi\left(8 h^{-1} \mathrm{Mpc}\right)$. Direct comparison of the $\left\langle\delta_{8}\right\rangle$ and $\xi(r)$ requires a correction for the presence of redshift distortions due to infall and "fingers of God".

On the $1 h^{-1}$ Mpc scale, the estimate of environment overdensity $\delta_{1}$ is a deprojected angular correlation function. Around each spectroscopic target galaxy, galaxies are counted in the SDSS imaging in the magnitude range corresponding to $M^{*} \pm 1$ mag (passivelyevolved and $K$-corrected as for an early-type galaxy) and within $5 h^{-1} \mathrm{Mpc}$ (transverse, proper; e.g., Hogg 1999) at the spectroscopic galaxy redshift. The count is weighted so as to recover the estimated overdensity averaged over a spherical three-dimensional Gaussian window $e^{-r^{2} / 2 a^{2}}$ with a radius of $a=1 h^{-1} \mathrm{Mpc}$ (proper). Details of the weighting and the method for correcting for the survey mask are given elsewhere (Eisenstein 2002). The results do not depend on an assumed model of the correlation function but do depend inversely on the normalization of the luminosity function at the redshift in question. One advantage of this method is that the density can be estimated with a volume-limited and yet reasonably dense set of galaxies, even at the furthest reaches of the spectroscopic catalog. Another advantage is that the estimator is not affected by redshift distortions. If the spatial correlation function $\xi(r)$ has the form $r^{-\gamma}$, then the mean overdensity around galaxies $\left\langle\delta_{1}\right\rangle$ is $(2 / \sqrt{\pi}) \Gamma([3-\gamma] / 2) \xi\left(1 h^{-1} \mathrm{Mpc}\right)$.

The two overdensity estimators $\delta_{1}$ and $\delta_{8}$ are very different; the deprojection technique is not possible on the large scale because of survey geometry constraints, and the redshift-angle 
sphere technique is not possible on the small scale because of peculiar velocities. Of course, that different estimators with different systematics are used on the two different scales makes their inter-comparison informative.

\section{Results}

Fig 1 shows the mean dependencies of overdensity on luminosity and color separately. Both show monotonic trends, with redder and more luminous galaxies, on average, in higher density regions. In each panel the mean is weighted by the inverse selection volumes $1 / V_{\max }$.

Panel (a) of Fig 2 shows the distribution of galaxies in the sample in the luminosity-color plane. The "red sequence" of old stellar populations, i.e., early-type galaxies, is visible. We will refer to galaxies on this sequence as "red" and those bluer as "blue." The mode of the luminosity distribution is more luminous than $L^{*}$. This is due to the classical Malmquist bias: more luminous galaxies are visible over a much larger volume and are therefore overrepresented in the sample. Taking means weighted by $1 / V_{\max }$ compensates for this bias.

Panels (b) and (c) of Fig 2 show the weighted mean environment overdensities $\left\langle\delta_{8}\right\rangle$ and $\left\langle\delta_{1}\right\rangle$ computed in a sliding box of luminosity width $\Delta M_{0.1} i_{i}=0.5 \mathrm{mag}$ and color width $\Delta^{0.1}(g-r)=0.15 \mathrm{mag}$, at all colors and magnitudes at which the sliding box contains at least 200 galaxies. The trends of the two different density estimators are remarkably similar:

(1) Galaxies of every color and luminosity are, on average, in overdense regions $(\delta>0)$. There are no unclustered or void populations that can be isolated by color and luminosity, in the color and luminosity ranges considered here.

(2) Very luminous $\left(L>3 L^{*}\right)$ red galaxies are, on average, in more overdense regions than the average galaxy. This is not surprising, given that many clusters contain extremely luminous galaxies (e.g., Beers \& Geller 1983).

(3) Faint $\left(L<(1 / 3) L^{*}\right)$ red galaxies are also, on average, in more overdense regions than the average galaxy. This may be related to the observation that the luminosity function appears more dwarf-rich in clusters than in the field (e.g., Driver et al. 1994; Bernstein et al. 1995; de Propris et al. 1995; Trentham \& Hodgkin 2002).

(4) The mean overdensity of blue galaxies is independent of luminosity and increases with color, at least at luminosities $L<3 L^{*}$. That overdensity is related to color reflects the color-morphology and density-morphology relations (Dressler 1980; Postman \& Geller 1984; Roberts \& Haynes 1994). 


\section{Discussion}

We have shown that blue galaxies, i.e., galaxies bluer than the red sequence of old stellar populations, exhibit no correlation between their luminosities and the overdensity of their environments, at fixed color. The class of blue galaxies includes most spirals and irregulars.

On Fig 2 a line of (roughly) constant stellar mass is shown, derived by converting relations derived in the $B$ and $R$ bands (Bell \& de Jong 2001); the conversion is straightforward because $B$ and $R$ are very similar to ${ }^{0.1} \mathrm{~g}$ and ${ }^{0.1} i$. The line of constant mass is close to a line of constant luminosity. Similarly, a line of constant star-formation history is close to a line of constant color, with a small slope arising from the luminosity-metallicity relation (e.g., Vila-Costas \& Edmunds 1992; Zaritsky et al. 1994; Ryder 1995). Fig 2 shows that, for spiral galaxies, mean overdensity is monotonically related to star-formation history. That there is a strong relationship between star-formation history and environment has been suggested before, both theoretically (eg, Kauffmann et al. 1999; Benson et al. 2000) and observationally (Balogh et al. 2001; Gomez et al. 2002). What is more remarkable is that, for galaxies with the colors of spirals, at fixed star-formation history, overdensity does not appear to depend on stellar mass at all.

The reddest galaxies show strong trends in overdensity at both the luminous and faint extremes. The former trend indicates that the very most luminous galaxies lie in the very largest overdensities; probably this is related to the fact that giant galaxies lie near the centers of clusters. The latter trend suggests that though high-density regions are expected to be rich in dwarf galaxies, those dwarfs will, by and large, be much redder than typical galaxies of their luminosity (Norberg et al. 2002; Zehavi et al, in preparation). That red galaxies have a minimum in their mean overdensity at luminosities near $L^{*}$ could be partly due to the specific mixture of galaxy types at those luminosities; there is a large overlap with the distribution of spirals, and the red population contains a large number of Sa galaxies at those luminosities (e.g., Strateva et al. 2001).

At one point it was thought that dwarf galaxies might "fill the voids," making them an underdense population in the mean. Our results rule this out, at least for dwarf galaxies selected by color and luminosity in the ranges considered here.

We thank Andreas Berlind, Douglas Finkbeiner, Jill Knapp, Ravi Sheth, Roman Scoccimarro, Iskra Strateva, and Simon White for useful discussions and software. This research made use of the NASA Astrophysics Data System. MRB and DWH are partially supported by NASA (grant NAG5-11669) and NSF (grant PHY-0101738). DJE is supported by NSF (grant AST-0098577) and by an Alfred P. Sloan Research Fellowship. 
Funding for the creation and distribution of the SDSS has been provided by the Alfred P. Sloan Foundation, the Participating Institutions, the National Aeronautics and Space Administration, the National Science Foundation, the U.S. Department of Energy, the Japanese Monbukagakusho, and the Max Planck Society. The SDSS Web site is http://www . sdss . org/.

The SDSS is managed by the Astrophysical Research Consortium (ARC) for the Participating Institutions. The Participating Institutions are The University of Chicago, Fermilab, the Institute for Advanced Study, the Japan Participation Group, The Johns Hopkins University, Los Alamos National Laboratory, the Max-Planck-Institute for Astronomy (MPIA), the Max-Planck-Institute for Astrophysics (MPA), New Mexico State University, University of Pittsburgh, Princeton University, the United States Naval Observatory, and the University of Washington.

\section{REFERENCES}

Balogh, M. L., Christlein, D., Zabludoff, A. I., \& Zaritsky, D. 2001, ApJ, 557, 117

Beers, T. C. \& Geller, M. J. 1983, ApJ, 274, 491

Bell, E. F. \& de Jong, R. S. 2001, ApJ, 550, 212

Benson, A. J., Baugh, C. M., Cole, S., Frenk, C. S., \& Lacey, C. G. 2000, MNRAS, 316, 107

Bernstein, G. M., Nichol, R. C., Tyson, J. A., Ulmer, M. P., \& Wittman, D. 1995, AJ, 110, 1507

Blanton, M. R., Brinkmann, J., Csabai, I., Doi, M., Eisenstein, D. J., Fukugita, M., Gunn, J. E., Hogg, D. W., \& Schlegel, D. J. 2002a, AJ, in press (astro-ph/0205243)

Blanton, M. R., Lin, H., Lupton, R. H., Maley, F. M., Young, N., Zehavi, I., \& J., L. 2002b, AJ, in press (astro-ph/0105535)

Blanton, M. R. et al. 2002c, ApJ, submitted (astro-ph/0209479)

Blanton, M. R. et al. 2002d, ApJ, submitted (astro-ph/0210215)

Davis, M. \& Geller, M. J. 1976, ApJ, 208, 13

de Propris, R., Pritchet, C. J., Harris, W. E., \& McClure, R. D. 1995, ApJ, 450, 534

Dressler, A. 1980, ApJ, 236, 351 
Driver, S. P., Phillipps, S., Davies, J. I., Morgan, I., \& Disney, M. J. 1994, MNRAS, 268, 393

Eisenstein, D. J. 2002, ApJ, submitted (astro-ph/0212084)

Eisenstein, D. J. et al. 2001, AJ, 122, 2267

Eisenstein, D. J. et al. 2002, AJ, submitted (astro-ph/0212087)

Fukugita, M., Ichikawa, T., Gunn, J. E., Doi, M., Shimasaku, K., \& Schneider, D. P. 1996, AJ, 111, 1748

Gomez, P. et al. 2002, ApJ, in press (astro-ph/0210193)

Gunn, J. E., Carr, M. A., Rockosi, C. M., Sekiguchi, M., et al. 1998, AJ, 116, 3040

Hogg, D. W. 1999, astro-ph/9905116

Kauffmann, G., Colberg, J. M., Diaferio, A., \& M., W. S. D. 1999, MNRAS, 303, 188

Kauffmann, G., Nusser, A., \& Steinmetz, M. 1997, MNRAS, 286, 795

Kennicutt, R. C. 1983, AJ, 88, 483

Kormendy, J. \& Djorgovski, S. 1989, ARA\&A, 27, 235

Lemson, G. \& Kauffmann, G. 1999, MNRAS, 302, 111

Lewis, I. et al. 2002, MNRAS, 334, 673

Lupton, R. H., Gunn, J. E., Ivezić, Z., Knapp, G. R., Kent, S., \& Yasuda, N. 2001, in ASP Conf. Ser. 238: Astronomical Data Analysis Software and Systems X, Vol. 10, 269-??

Martínez, H. J., Zandivarez, A., Domínguez, M., Merchán, M. E., \& Lambas, D. G. 2002, MNRAS, 333, L31

Mo, H. J., McGaugh, S. S., \& Bothun, G. D. 1994, MNRAS, 267, 129

Mo, H. J. \& White, S. D. M. 1996, MNRAS, 282, 347

Norberg, P. et al. 2002, MNRAS, 332, 827

Park, C., Vogeley, M. S., Geller, M. J., \& Huchra, J. P. 1994, ApJ, 431, 569

Petrosian, V. 1976, ApJ, 209, L1 
Pier, J. R., A., M. J., Hindsley, R. B., Hennessy, G. S., Kent, S. M., Lupton, R. H., \& Ivezić, Z. 2002, AJ, submitted

Postman, M. \& Geller, M. 1984, ApJ, 281, 95

Richards, G. et al. 2002, AJ, 123, 2945

Roberts, M. S. \& Haynes, M. P. 1994, ARA\&A, 32, 115

Ryder, S. D. 1995, ApJ, 444, 610

Schlegel, D. J., Finkbeiner, D. P., \& Davis, M. 1998, ApJ, 500, 525

Smith, J. A., Tucker, D. L., et al. 2002, AJ, 123, 2121

Stoughton, C. et al. 2002, AJ, 123, 485

Strateva, I. et al. 2001, AJ, 122, 1861

Strauss, M. A. et al. 2002, AJ, 124, 1810

Tammann, G. A., Yahil, A., \& Sandage, A. 1979, ApJ, 234, 775

Trentham, N. \& Hodgkin, S. 2002, MNRAS, 333, 423

Vila-Costas, M. B. \& Edmunds, M. G. 1992, MNRAS, 259, 121

York, D. et al. 2000, AJ, 120, 1579

Zaritsky, D., Kennicutt, R. C., \& Huchra, J. P. 1994, ApJ, 420, 87

Zehavi, I. et al. 2002, ApJ, 571, 172 

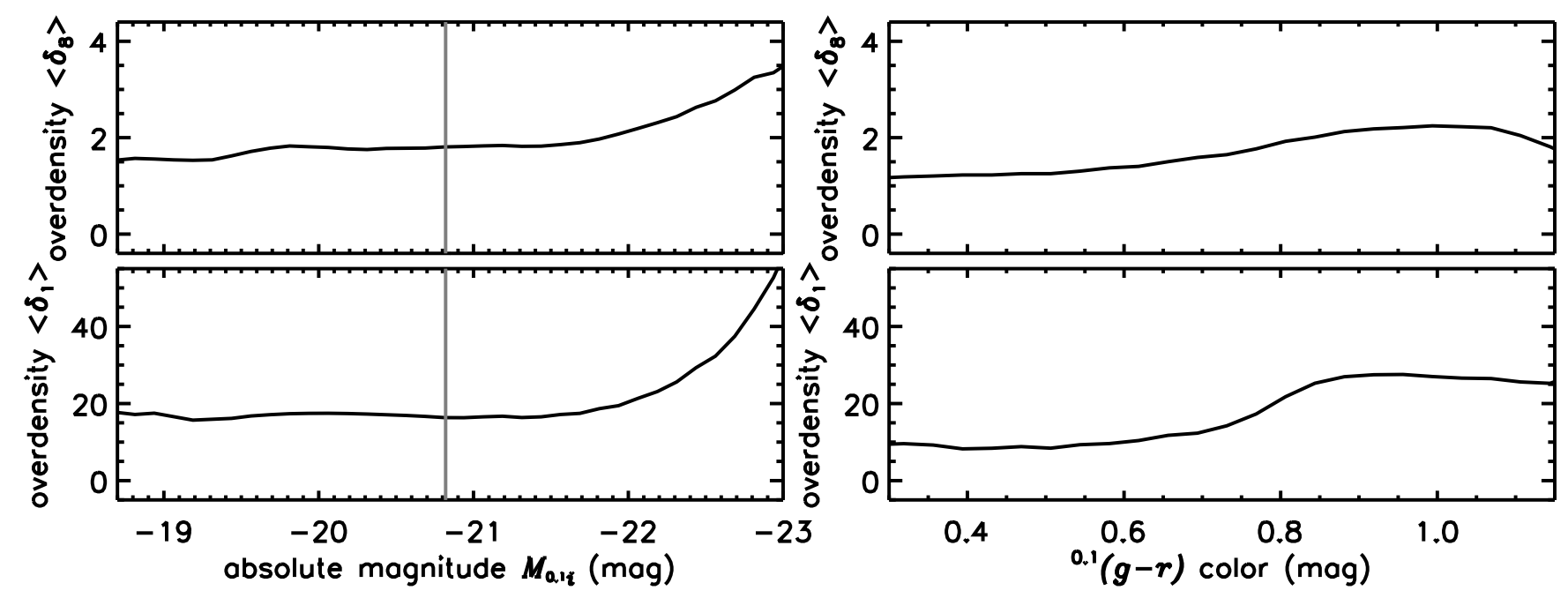

Fig. 1. - The $1 / V_{\max }$-weighted mean galaxy environment overdensities $\left\langle\delta_{8}\right\rangle$ and $\left\langle\delta_{1}\right\rangle$ in $8 h^{-1} \mathrm{Mpc}$ and $1 h^{-1} \mathrm{Mpc}$ spheres (see text) computed in a sliding box of luminosity width $\Delta M_{0.1_{i}}=0.5 \mathrm{mag}$ (absolute magnitude plots) or in a sliding box of color width $\Delta^{0.1}(g-r)=$ 0.15 mag (color plots). A vertical line shows the characteristic luminosity $L^{*}$ (Blanton et al. 2002d). 

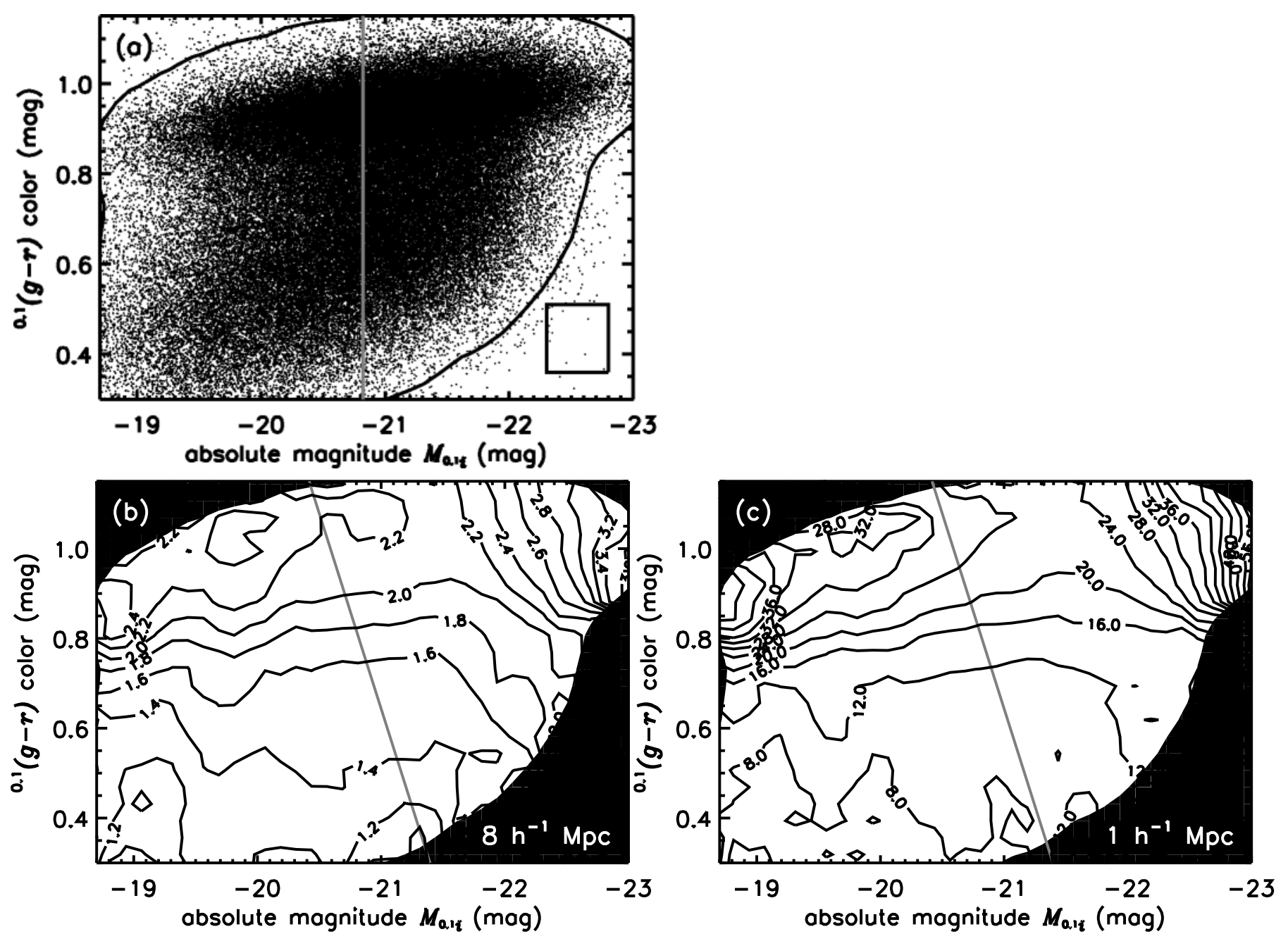

Fig. 2.- Panel (a) shows the luminosity and color of each galaxy in the sample. A vertical grey line shows the characteristic luminosity $L^{*}$ (Blanton et al. 2002d). Panel (b) shows the $1 / V_{\max }$-weighted mean galaxy environment overdensity $\left\langle\delta_{8}\right\rangle$ in $8 h^{-1}$ Mpc spheres (see text) computed in a sliding, rectangular box of luminosity width $\Delta M_{0.1_{i}}=0.5$ mag and color height $\Delta^{0.1}(g-r)=0.15 \mathrm{mag}$. The box size and shape is shown on the lower right corner of panel (a). The grey line indicates, roughly, a locus of constant total stellar mass (Bell \& de Jong 2001). Panel (c) shows the weighted mean overdensity $\left\langle\delta_{1}\right\rangle$ in $1 h^{-1} \mathrm{Mpc}$ spheres (see text). Note that different kinds of overdensity estimators are used for the two different length scales. The mean environment overdensity is not shown shown at colors and luminosities at which there are $<200$ galaxies inside the box; this region is indicated with a border on panel (a). 